\title{
Effects of video frame averaging, smoothing and edge enhancement on the accuracy and precision of quantitative coronary arteriography
}

\author{
Kevin M. Kavanaugh, Ibraim M.F. Pinto, Mark J. McGillem, Scott F. DeBoe, G.B. John Mancini \\ University of Michigan School of Medicine and the Ann Arbor Veterans Administration Medical Center, \\ Ann Arbor, Michigan, USA
}

\begin{abstract}
Digital analysis of cine film provides numerous options for altering images by frame averaging or filtering algorithms that either smooth or enhance edges. While these may subjectively enhance image quality, there is no uniformity in their use among laboratories and effects on quantitative coronary analysis may not be ideal. To determine which processing algorithms might help or hinder quantitative coronary arteriography, cine film images of precision drilled stenotic cylinders $(0.83$ to $1.83 \mathrm{~mm}$ diameter $)$ implanted in dog coronary arteries were analyzed with and without such algorithms. Video frame averaging of 1 to 49 frames had no effect on measures of accuracy (mean differences) but precision (standard deviation of mean differences) was improved from 0.23 to $0.17 \mathrm{~mm}(\mathrm{p}<0.05)$ with video averaging of $\geq 25$ frames. Edge enhancement filtering algorithms resulted in slight deterioration of accuracy and precision and smoothing filtering algorithms caused modest improvements in these parameters; however, these changes were not significantly different from unprocessed images. Using edge enhancement filtering algorithms, accuracy was significantly worse $(-0.27 \mathrm{~mm})$ compared to a smoothing filter enhancement algorithm $(-0.08 \mathrm{~mm}, \mathrm{p}<0.001)$. The combination of video averaging and smoothing algorithms had no additional beneficial effects. Thus, precision of quantitative coronary analysis of cine film can be optimized by appropriate video averaging. Edge enhancement filtering algorithms should be avoided whereas smoothing filter enhancement algorithms may improve accuracy.
\end{abstract}

\section{Introduction}

Quantitative coronary arteriography is useful in reliably assessing the extent of coronary disease [1-3]. Video noise can be reduced by multiple digitizations of video frames and then averaging the output. Edge enhancement and smoothing algorithms have been shown to improve the qualitative appearances of a variety of digitized images [4]. Reiber and others have argued for greater uniformity in quantitative arterial analyses, yet these processing techniques are used erratically or differently in many laboratories. Moreover, effects of image manipulation on the results of in vivo quantitative coronary analysis have not been previously reported. The purpose of this study was to determine the optimal number of video frame averages to reduce video noise and to test available smoothing and edge enhancement algorithms to determine if their implementation improves or deteriorates automatic lesion quantitation. This study was undertaken with images from an in vivo experiment so that results could be interpreted with respect to true accuracy and precision in a model that is relevant to clinical use of quantitative arteriography.

\section{Methods}

Seven mongrel dogs had a total of nine precision 
drilled, radiolucent nylon stenosing cylinders (Poly C Co., Ann Arbor, MI) implanted in their proximal coronary arteries (left anterior descending and/or left circumflex) as previously described [1]. The distal $2 \mathrm{~mm}$ of the cylinders were drilled to produce lumen diameters ranging from 0.71 to $1.83 \mathrm{~mm}$. Biplane cineangiograms were then obtained with a standard system (Philips Optimus M200, Eindhoven, The Netherlands) in projections that optimized separation of the stenotic cylinders from surrounding vessels. The radiographic input signal was kept constant (fixed $\mathrm{kVp}, \mathrm{MA}$, and pulse width $x$-ray exposure). Care was taken to position both the stenotic area and the angiographic catheter within the central portion of the radiographic field to minimize pincushion distortion. A $12.5 \mathrm{~cm}$ field of view and a small focal spot size $(0.6 \mathrm{~mm}$, nominal) were used. Images were acquired at 30 frames/sec. Images that optimally showed the lesions were projected on a Vanguard viewer (Model XRP 15, Melville, NY), which was optically coupled to a video camera. With $2.4: 1$ optical magnification, the video signal correponding to a subregion of the $35 \mathrm{~mm}$ frame was digitized at $512 \times 512 \times 8$ bit resolution. The optimal light intensity for digitization at the $2.4: 1$ magnification was determined for the region of the image containing the catheter segment and the stenotic area. Video frame averaging or application of filters (see below) was performed after this step. The optical densities of interest were on the linear portion of the sensitometric curve of the film (Kodak CFR) ensured by routinely analyzing a density stepwedge.

Film images were preprocessed before quantitative analysis. All images were subjected to gray scale expansion to fill the full 8 bit dynamic range of the digital radiographic system. This preprocessing step is fully automatic. Digitized film images were then magnified by a factor of 2 achieved by bilinear pixel interpolation using the system's array processor. Although this digital magnification does not improve spatial sampling density of the electronic imaging methods, it does provide additional precision in the analysis techniques of the quantitative program. The final overall magnification of the digitized film images was $\times 4.8$, with an effective pixel resolution of $2458 \times 2458$. This magnification was determined experimentally to optimize the quantitative analysis of film images [5].

Each image of the 9 intracoronary stenosing cylinders were averaged $1,4,9,16,25,36$ and 49 times prior to storage and analysis. This process was undertaken 6 times for all the images to increase the number of observations so as to increase the statistical power of the analysis.

During a separate digitization of cineangiograms, each of 9 smoothing and edge enhancement algorithms, or none, were applied to the images prior to analysis. All 9 filters used are $3 \times 3$ convolutions with the coefficient matrix:

$\begin{array}{lll}\mathrm{C} 3 & \mathrm{C} 2 & \mathrm{C} 3 \\ \mathrm{C} 2 & \mathrm{C} 1 & \mathrm{C} 2 \\ \mathrm{C} 3 & \mathrm{C} 2 & \mathrm{C} 3\end{array}$

This coefficient matrix is then applied to the pixel matrix:

$\begin{array}{lll}\text { P1 } & \text { P2 } & \text { P3 } \\ \text { P4 } & \text { P5 } & \text { P6 } \\ \text { P7 } & \text { P8 } & \text { P9 }\end{array}$

These matrices are used to produce the new pixel Q5 corresponding to P5 using the formula:

$$
\begin{aligned}
& Q 5=C 1 P 5+C 2(P 2+P 4+P 6+P 8)+ \\
& C 3(P 1+P 3+P 7+P 9)
\end{aligned}
$$

The unity gain formula is:

$$
C 1+4 C 2+4 C 3=1
$$

The actual coefficients are shown in Table 1. Essentially, edge enhancement options increase intensity differences between P5 and its neighbors while the smoothing options decrease these differences. Thus, edge enhancements tend to make the edges stand out whereas the smoothing options tend to blur them. These algorithms were those that are available on the commercial system used in this project. An attempt to develop different filters or optimal filters was not undertaken in this study.

Images were analyzed with a previously de- 
scribed automatic coronary quantitation program [5]. Briefly, the operator chooses a circular region for analysis by first positioning a lightpen cursor over the arterial lesion and then adjusting the size of the circular region to encompass the desired segment of artery to be analyzed. The software then proceeds without further operator intervention. The centerline of the arterial segment within the analysis region is determined by analyzing circular pixel density profiles of decreasing radii, with use of simple signal processing techniques to locate the angular positions of the proximal and distal portions of the arterial segment at each radius. When the radius approaches zero, the entire arterial centerline has been calculated. Linear density profiles perpendicular to the arterial centerline are extracted over the entire length of the arterial segment. Edge points are found by analyzing the linear density profiles in 2 passes. Initial edge points are found by noting the density of points at the first and second derivatives of each perpendicular density profile and then determining the location of the points that fall at a value of $75 \%$ of the difference between the densities at these derivative extrema (i.e., weighted toward the first derivative extrema). This method was found to give optimal accuracy and precision of measurement of radiographic phantoms in the 0.5 to $5 \mathrm{~mm}$ diameter range [5]. These initial gradient-determined edge points are then examined for spatial continuity and outliers are discarded. That is, because of radiographic or film noise and adjacent structures common in in vivo imaging, the initial gradient-determined edge points sometimes fail to form a continuous edge contour. Rather than smoothing the resulting noisy edge contours, spurious edge points are discarded with application of a spatial continuity criterion. Should a given initial edge point lie more than an empirically determined (four-pixel) distance from its neighbors, it is marked as not lying on the true arterial edge and discarded. During the second pass, the gray scale densities of initial edge points on either side of the discarded points are then used to determine the final edge points by linear interpolation from the densities at neighboring, valid edge points.

The geometric diameter at any point along the centerline is the distance along each perpendicular profile between edge points on opposite sides of the artery. Calibration is achieved by measuring a magnification factor based on the known size of the angiographic catheter. In this study, calibrations ranged from 0.0560 to $0.0991 \mathrm{~mm} / \mathrm{pixel}$. The final computer output consists of the arterial image with edges and centerline and plots of geometric diameter (calibrated with reference to the known diameter of the angiographic catheter) and maximal percent diameter stenosis. The minimum diameter was recorded and studied in this project.

Output from the quantitative coronary analysis of the stenosis were then compared to known diameters for each respective cylinder. The mean difference (accuracy) and the standard deviation of the mean difference (precision) along with the mean absolute difference were calculated for each experimental condition. Statistical analysis of values for mean difference and mean absolute difference was carried out by analysis of variance. The

Tablel.

\begin{tabular}{llcc}
\hline & $\mathrm{C} 1$ & $\mathrm{C} 2$ & $\mathrm{C} 3$ \\
\hline EDGE 1 & 1.38627017 & -0.05657 & -0.04 \\
EDGE 2 & 1.67598009 & -0.09899 & -0.07 \\
EDGE 3 & 2.18778969 & -0.17395 & -0.123 \\
EDGE 4 & 3.06657004 & -0.30264002 & -0.214 \\
EDGE 5 & 4.62131977 & -0.53032994 & -0.375 \\
SMOOTH 1 & 0.80686003 & 0.0282 & 0.02 \\
SMOOTH 2 & 0.42059001 & 0.08485 & 0.06 \\
SMOOTH 3 & 0.13088 & 0.12728001 & 0.09 \\
SMOOTH 4 & 0.11111 & 0.11111 & 0.11111 \\
\hline
\end{tabular}


analysis for video frame averaging was a 2 factor mixed effects non-additive model [6], the filtering algorithm analysis used a repeated measures model [6]. If overall significant differences were found, Newman Keuls simultaneous pairwise comparisons were used [7]. Measures of variability are represented as the standard deviation of the mean difference and statistically significant differences were assessed by use of a Pitman test for correlated variances while using the Bonferroni technique to control for repeated observations [8]. Due to the small range of diameters of the phantoms, analyses were not stratified according to diameter sizes.

\section{Results}

Video averaging did not significantly improve the accuracy of the quantitative analysis as assessed by examination of the mean difference. However, precision (standard deviation of the mean difference) was significantly enhanced at 25 video frame averages (VFA) when compared to 9 VFA $(\mathrm{p}<$ $0.05)$. At $25 \mathrm{VFA}$, the precision was $0.17 \mathrm{~mm}$ compared to $0.23 \mathrm{~mm}$ with no averaging. Mean absolute difference was also enhanced with 25 VFA when compared to 1 or 9 VFA $(p<0.05)$. Averaging with 36 or 49 digitizations resulted in no further improvement in precision or absolute mean difference (Fig. 1).

The effects of nine filtering algorithms on image analysis were compared to the quantitative results obtained from images that were not subjected to video frame averaging or any form of filtration. The edge enhancement algorithms caused slight deterioration of accuracy and precision, though these were not statistically significant. Smoothing filtering algorithms caused a slight improvement in accuracy and mean absolute differences when compared to the unprocessed images. Again, however, the improvements were not statistically significant. When the smoothing algorithms were compared directly with the edge enhancement algorithms, a significant improvement in accuracy was found $(-0.08$ vs $-0.27 \mathrm{~mm}$, respectively, $\mathrm{p}<0.001$, Fig. 2).

\section{Discussion}

Use of quantitative coronary analysis offers advantages over visual estimations of coronary artery lesion severity. There is large inter- and intra-observer variability with visual evaluation of coronary cineangiograms [9-13] which can be minimized by computerized methods $[1,5,14,15-18]$. Clinical studies evaluating various treatment regimens have mandated a more reproducible method for evaluating coronary disease and, thus, quantitative coronary analysis has gained widespread implementation. Methods designed to enhance the diagnostic accuracy of automated quantitative coronary analysis such as video frame averaging and filtering algorithms are commercially available and are frequently implemented. Such manipulations on quantitative coronary analysis have not been rigorously evaluated, however, and their use is not uniform.

The present study investigated the effects of video frame averaging and filtering algorithms on the accuracy and precision of automated quantitative coronary analysis. These image processing utilities are available on most quantitative coronary analysis systems, however, to date there is no information on the effects these processes have on in vivo lesion measurement. By comparing results of automated lesion measurements with known stenosis diameters, we draw the following conclusions:

1) The precision of the automated quantitative coronary analysis used in this study is optimized by appropriate video frame averaging.

2) Edge filter enhancement algorithms resulted in no improvement and perhaps some deterioration in the performance of quantitative coronary analysis and thus should be avoided.

3) Smoothing algorithms resulted in some enhanced accuracy when compared with the edge filtering algorithms and may have some benefit for quantitative coronary analysis.

The signal-to-noise ratio of an image produced by averaging multiple images is greater than the signal-to-noise ratio of the individual images averaged to produce it. The theoretical signal-to-noise ratio of an image produced by averaging $\mathrm{N}$ images 


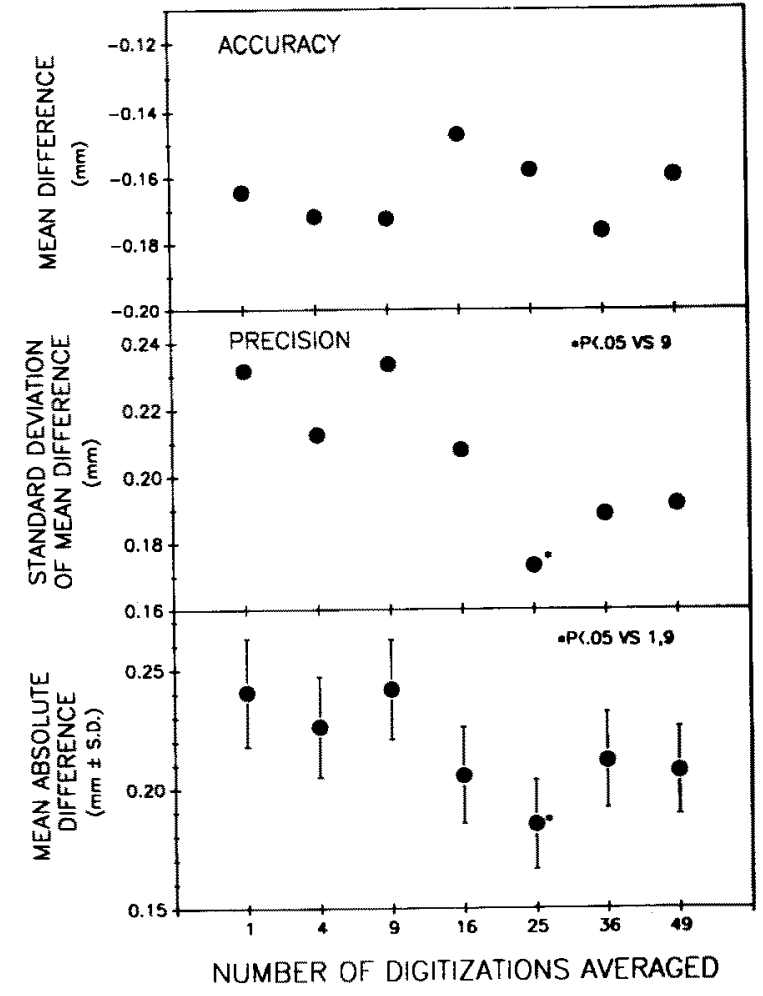

Fig. 1. Effects of video frame averaging on accuracy (mean difference), precision (standard deviation of mean difference) and mean absolute differences are shown. The $\mathrm{x}$-axis designates the number of frames averaged to produce a single image subjected to quantitation. Although accuracy was not affected, optimal results for precision and mean absolute differences were obtained by averaging 25 frames.

is approximately the square root of $\mathrm{N}$ times that of each of the individual images [19]. The present study revealed that 25 digitizations resulted in a significant improvement in the precision of the measurement and that further averaging was of no additional benefit. While the results in this study are based upon relatively high quality images and the results are modest, Fig. 3 shows an example of the effects of video averaging when initial image quality is poor. While this theoretically reflects predominantly the reduction of video noise, it is clear from Fig. 3 that the effects of this process are also modulated by the intrinsic initial quality of the film to be analyzed. Without video averaging, this image could not be analyzed by the automated technique without excessive editing. Excessive ed-

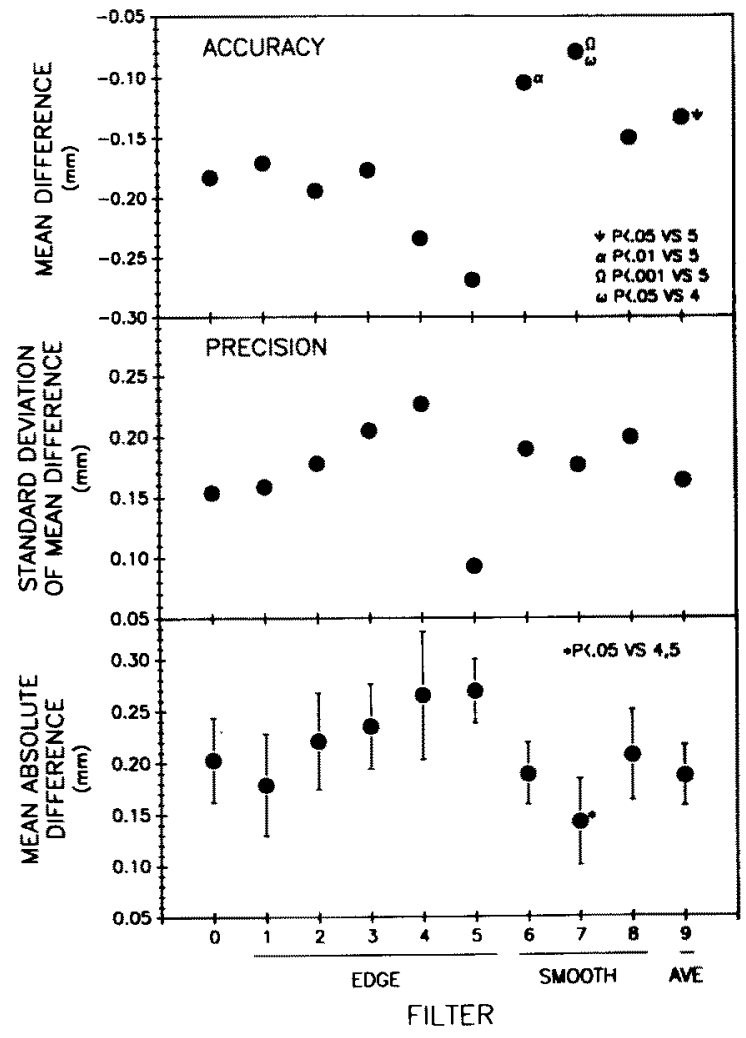

Fig. 2. Effects of filtering algorithms are shown in the same format as Fig. 1. The abscissa designates edge enhancement filters (EDGE, 1-5), smoothing filters (SMOOTH, 6-8) and an averaging filter (AVE, 9). The designation ' 0 ' refers to unfiltered results. Although precision was unaffected, accuracy and mean absolute difference measures were better using smoothing algorithms compared to edge enhancement algorithms.

iting essentially nullifies the attractiveness of automatic edge detection programs. The pre-processing substantially minimizes the need for editing the automatically determined edges and thereby substantially decreases the work involved in high volume laboratories committed to quantitation of cineangiograms. It should also enhance reproducibility since observer interaction is minimized. Such video frame pre-processing also allows greater applicability of the automated analysis when quantitating films that are not of excellent quality. That is, fewer films are deamed unsuitable for quantitation. These are practical and important issues with implications for performance of studies involving many centers and many patients. Ac- 

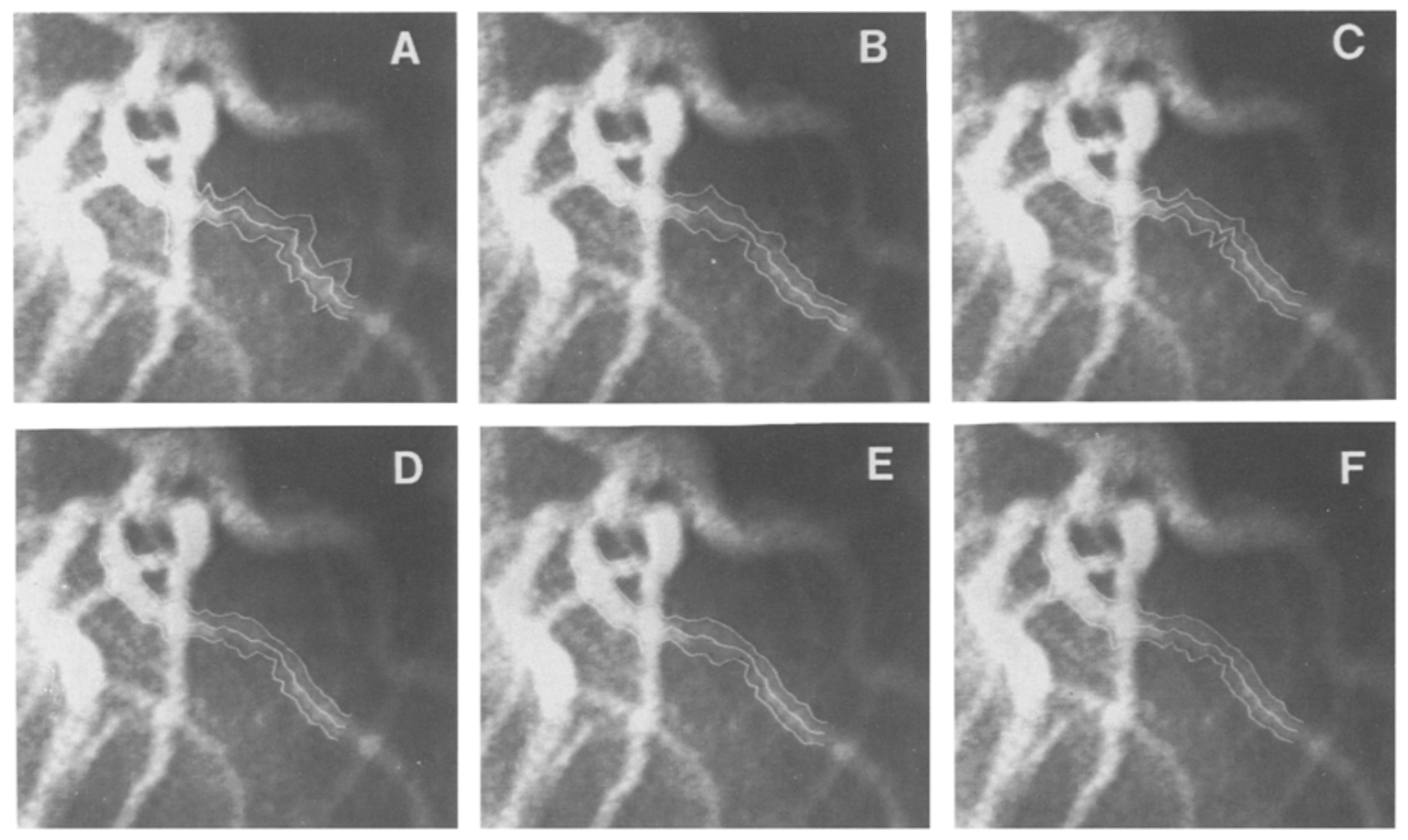

Fig. 3. Effects of video frame averaging on the acceptability of automatic edge detection are demonstrated. This frame was obtained from a suboptimal clinical cineangiogram. Edge detection is erratic in Panel $A$ (single frame) and improves progressively as video averaging is employed. The number of frames used were $4,9,25,36$ and 49 for panels $B, C, D, E$ and F respectively. Panels D, E and F show the most acceptable automatic edge tracking results.

cordingly, our policy, designed to maintain uniformity and enhance robustness of edge tracking, is to routinely use 25 frames of video averaging when analyzing film.

The use of digital filtering algorithms has been implemented as a means of improving signal-tonoise ratios and enhancing the qualitative appearance of the image. Such filtering of digitally acquired images has been suggested to improve the diagnostic, qualitative content of radiographic studies. Kimme-Smith et al. [4] reported the use of digital filters as having some improvement on the spatial or contrast resolution of digitally subtracted renal angiograms. However, diagnostic accuracy was not improved with the use of these filters. The qualitative appearance of digitized images subjected to these filtering algorithms is sometimes dramatic; what effect there is on the actual quantitative coronary analysis measurements is not known. Moreover, since such filters are available on com- mercial systems that also provide options for automatic edge detection, the temptation exists to combine the two. We set out to determine the effects on quantitation when such filters, which are of subjective value, are used in quantitative analyses. Our results suggest that edge enhancement filters offer no improvement in quantitative analyses and they may be deleterious. Smoothing enhancement filters may improve the accuracy to some degree and may be helpful in some images.

It should be recognized that these experiments should not be extrapolated literally to the performance of other systems of quantitative arteriography. The results do suggest that potential effects should be scrutinized in each laboratory so that the process of analysis can be standardized and optimized. 


\section{References}

1. Mancini GBJ, Simon SB, McGillem MJ, LeFree MT, Friedman HZ, Vogel RA. Automated quantitative coronary arteriography: morphologic and physiologic validation in vivo of a rapid digital angiographic method. Circulation 1987; 75: 452-60.

2. Brown BG, Bolson EL, Dodge HT. Quantitative computer techniques for analyzing coronary arteriograms. Prog Cardiovasc Dis 1986; 28: 403.

3. Reiber JHC, Kooijman CJ, Slager CJ, Gerbrands JJ, Schuurbiers JCH, Den Boer A, Wijns W, Serruys PW. Computer assisted analysis of the severity of obstructions from coronary cineangiograms: a methodological review. Automedica 1984; 5: 219.

4. Kimme-Smith C, Gomes AS, Cochran ST, Barbaric ZL, Lois JF. Diagnostic effects of edge sharpening filtration and magnification on digitally subtracted renal images. Med Phys 1986; 13: 850-6.

5. LeFree MT, Simon SB, Mancini GBJ, Vogel RA. Digital radiographic assessment of coronary arterial diameter and videodensitometric crossectional area. Proc SPIE 1986; 626: 334 .

6. Neter J, Wasserman W. Applied Linear Statistical Models. Homewood, Illinois, Richard D Irwin, Inc, 1974; 616-27.

7. Miller RG. Simultaneous Statistical Inference. 2nd Edition. New York, New York. Springer-Verlag, 1985; 81-90.

8. Snedecor GW, Cochran WG. Statistical Methods. 6th Edition. Ames, Iowa. Iowa State University Press, 1972; 195-7.

9. Fisher LD, Judkins MP, Cameron LJ, Swaye P, Ryan T, Maynard C, Bourassa M, Kennedy JW, Gosselin A, Kemp H, Faxon D, Wexler L, Davis KB. Reproducibility of coronary arteriographic reading in the Coronary Artery Surgery Study (CASS). Cathet Cardiovasc Diagn 1982; 8: 565-75.

10. Zir LM, Miller SW, Dinsmore E, Gilbert JP, Harthorne JW. Inter-observer variability in coronary angiography. Circulation 1976; 53: 627-32.

11. Arnett EN, Isner JM, Redwood DR, Kent KM, Baker WP, Ackerstein J, Roberts WC. Coronary artery narrowing in coronary heart disease: comparison of cineangiographic and necropsy findings. Ann Intern Med 1979; 91: 350-6.

12. DeRoun TA, Murray JA, Owen W. Variability in the analysis of coronary arteriograms. Circulation 1977; 55: 324-8.

13. Detre KM, Wright E, Murphy ML, Iakaro T. Observer agreement in evaluating coronary angiograms. Circulation $1975 ; 52: 979-86$.

14. Brown GB, Bolson E, Frimer M, Dodge HT. Quantitative coronary arteriography: estimation of dimensions, hemodynamic resistance and artheroma mass of coronary artery lesions using the arteriogram and digital computation. Circulation 1977 ; 55: 329-37.

15. Tobis J, Nalcioglu O, Iseri L, Johnston WD, Roeck WD, Castleman E, Bauer B, Montelli S, Henry WL. Detection and quantitation of coronary artery stenoses from digital subtraction angiograms compared with 35-millimeter film cineangiograms. Am J Cardiol 1984; 54: 489-96.

16. Vas R, Eigler N, Miyazonio C, Pfaff JM, Resser KJ, Weiss M, Nivatpumin T, Whiting J, Forrester J. Digital quantification eliminates intra-observer and inter-observer variability in the evaluation of coronary artery stenosis. Am J Cardiol 1985; 56: 718-23.

17. Nichols AB, Gabrieli CFO, Fenoglio JJ, Esser PD. Quantification of relative coronary arterial stenosis by cinevideodensitometric analysis of coronary arteriograms. Circulation 1984; 69: 512-22.

18. Spears JR, Sandor T, Als AV, Malagold M, Markis JE, Grossman W, Serur JR, Paulin S. Computerized image analysis for quantitative measurement of vessel diameter from cineangiograms. Circulation 1983; 68: 453-61.

19. Kruger RA, Mistretta CA, Houk TL, Riederer SJ, Shaw CG, Goodsitt MM, Crummy AB, Zwiebel W, Lancaster JC, Rowe GG, Flemming D. Computerized fluoroscopy in real time for noninvasive visualization of the cardiovascular system. Radiology 1979; 130: 49-57.

Address for offprints:

G.B.J. Mancini,

Chief, Cardiology Section (111A), Veterans Administration Medical Center, 2215 Fuller Road, Ann Arbor, Michigan 48105, USA 